\title{
Programa ACHEI: Atenção ao Chagásico com Educação Integral no Município de Maringá e Região Noroeste do Paraná, Brasil
}

\author{
The ACHEI Program: Chagas' D isease Awareness through Comprehensive \\ Education in the Municipality of Maringá, Northwest Paraná, Brasil
}

\author{
Silvana Marques de Araújo', Míriam Hitomi Andón, Dirceu José Cassarotti ${ }^{3}$, Daniela Cristina \\ Grégio D’Arce Mota², Silvana Maria Ribeiro Borges ${ }^{4}$ e Mônica Lúcia Gomes ${ }^{1}$
}

\begin{abstract}
Resumo Participaram deste trabalho 131 pacientes chagásicos procedentes de diferentes áreas endêmicas atendidos pelo Laboratório de Doença de Chagas da Universidade Estadual de Maringá (UEM). Estes pacientes descobriram que estavam infectados principalmente porque apresentaram sintomatologia $(58,1 \%)$ ou porque se dispuseram a doar sangue $(29,4 \%)$. Durante o tratamento etiológico contra o Trypanosoma cruzi, 45,2\% apresentaram queixas relacionadas a efeitos colaterais do benznidazol. Com base nestes dados foi criado o Programa ACHEl: Atenção ao Chagásico com Educação Integral. Implantado como um projeto de extensão, tem caráter multiprofissional/interdisciplinar. Foi programado com uma reunião mensal composta de uma primeira parte informativa específica, quando é também distribuído um folder explicando transmissão, sintomatologia e tratamento da doença de Çhagas. Na segunda parte é trabalhado o apoio psico-social, enfocando auto-estima e cidadania. É um espaço onde pacientes chagásicos podem compartilhar com seus iguais a dúvida sobre a sua qualidade de vida após o diagnóstico, o medo, a ansiedade, o estigma, o diagnóstico positivo e a convivência com a família/grupo social criando a oportunidade e ambiente para que cada paciente reflita sobre sua própria história e ações frente ao processo da doença.
\end{abstract}

Palavras-chaves: Atenção ao chagásico. Doença de Chagas. Assistência psicossocial. Trypanosoma cruzi.

\begin{abstract}
This study analyzes 131 chagasic patients from different endemic areas that came to the Chagas' disease laboratory at the Maringa State University. The subjects discovered they were infected principally because they presented symptoms (58\%) or donated blood (29.4\%). During etiologic treatment for Trypanosoma cruzi, $45.2 \%$ of benznidazole users complained of side effects. Based on these data, the ACHEI p rogram (Chagas' Disease Awareness through Comprehensive Education) was developed, which is a multiprofessional/interdisciplinary extension project. Monthly meetings are held that are divided into two parts: The first half of the meetings provide specific information, including the distribution of brochures explaining Chagas' disease transmission, symptoms and treatment. The second half of the meetings focuses on psychosocial assistance and includes topics such as self-esteem and personal responsibility. The meetings provide an environment for chagasic patients to share their concerns regarding post-diagnosis quality of life, fear, anxiety, stigma and family and social group relationships. In short, the meetings offer an opportunity for patients to reflect on their situation and to discover ways to deal with their disease.
\end{abstract}

Key-words: Chagas' disease awareness. Chagas' disease. Psycho-social assistance. Trypanosoma cruzi.

\footnotetext{
1. Departamento de Análises Clínicas, 2. Departamento de Psicologia, 3. Departamento de Medicina, 4. Unidade de Psicologia Aplicada, 5. Hemocentro da Universidade Estadual de Maringá, Maringá, PR.

Endereço para correspondência: Profa. Silvana Marques de Araújo. Departamento de Análises Clínicas, Setor de Parasitologia Básica/ Universidade Estadual de Maringá. Av. Colombo 5790, 87020-900 Maringá, PR, Brasil.

Telefax: 5544 261-4490

e-mail: marqueslima@wnet.com.br

Recebido para publicação em 16/7/2000.
} 
O Conselho Executivo da OMS, considerando o relatório do Diretor Geral sobre os progressos para a eliminação da transmissão da doença de Chagas na América Latina, em sua $51^{\text {a }}$ Assembléia de Saúde Mundial, declara seu compromisso com a meta de interrupção da transmissão desta protozoose por volta do ano $2010^{14}$. No Brasil, $80 \%$ da área endêmica da doença de Chagas estão sob vigilância, com taxas de infestação domiciliar inferiores a 5\%4. Da mesma forma, observa-se forte redução da infecção chagásica entre os doadores de sangue segundo dados da hemorrede pública ${ }^{6}$. É de esperar-se também a redução dos casos de transmissão congênita, uma vez que no prazo de 10 ou 20 anos não mais se observará mulheres chagásicas em idade fértil ${ }^{6}$. No entanto, existirá ainda um contingente de indivíduos já infectados e cientes ou não da sua condição, cujo atendimento converte-se em questão básica para as autoridades sanitárias, merecendo destaque especial candidatos à doação de sangue com sorologia positiva para o Trypanosoma cruzi ${ }^{8}$.

No Paraná a situação não é diferente. $O$ inquérito nacional para doença de Chagas $/ 1980^{2}$ aponta prevalência de $4 \%$ de reações positivas para o Trypanosoma cruzi para este estado. Por estimativa tinha-se 166.511 indivíduos infectados ${ }^{16}$. A doença de Chagas é de evolução crônica, característica de populações rurais de baixa renda e até o momento não conta com tratamento etiológico completamente eficaz e livre de efeitos colaterais importantes. É uma doença estigmatizante que pode desenvolver mudanças significativas na vida de seus portadores ${ }^{1}$. Este trabalho tem por objetivo relatar a experiência de implantação de um grupo de apoio psico-social para atendimento de pacientes chagásicos de Maringá e região Noroeste do Paraná além de descrever o perfil destes pacientes.

\section{MATERIAL E MÉTODOS}

Pacientes. Participaram deste trabalho pacientes triados em bancos de sangue, pacientes que recorreram ao SUS/Setor de Cardiologia de Maringá/PR e pacientes que foram diagnosticados em levantamentos na área rural de municípios do noroeste do Paraná e atendidos pelo laboratório de Doença de Chagas da Universidade Estadual de Maringá (UEM). Estes pacientes foram submetidos a um questionário para obter informações a cerca do sexo, idade, procedência do paciente, como o paciente descobriu a doença de Chagas e como ele suspeita ter se contaminado. Dados clínicos foram obtidos dos prontuários médicos arquivados no Centro Regional de Especialidades do Sistema Único de Saúde de Maringá (CRE/SUS - Maringá). Foi indicado o tratamento específico a pacientes com até 50 anos de idade, portadores de forma indeterminada ou formas cardíaca e digestiva incipientes. Acima desta idade, o tratamento foi indicado a pacientes que apresentassem boas condições de saúde geral e expressassem desejo de realizá-lo. A todos eram explicados os efeitos colaterais do medicamento e sua eficácia, providenciando a assinatura de um termo de concordância após a apresentação das informações acima referidas.

Implantação do Programa ACHEI. Optou-se pela implantação do programa como um projeto de extensão seguindo os trâmites normais de implantação de projetos dentro da Universidade Estadual de Maringá (UEM) na busca de viabilização rápida e desburocratizada no atendimento psicosocial a pacientes com sorologia positiva para a doença de Chagas. Desta forma estaria garantida a participação dos docentes do Laboratório de Doença de Chagas e do Departamento de Psicologia assim como dos profissionais da Unidade de Psicologia Aplicada/UEM e de acadêmicos das áreas de psicologia e da área da saúde. Estaria garantido também, espaço físico para a realização das reuniões com os pacientes. Embora pacientes triados pelo SUS tenham participado do programa, este projeto foi desenvolvido exclusivamente dentro da universidade sem nenhum envolvimento do SUS. A idéia era que se o projeto funcionasse bem como um piloto e mostrasse ser efetivo, poderia então ser levado à avaliação das autoridades sanitárias do município.

Divulgação do programa. Inicialmente, foram agendadas reuniões com os responsáveis pelos bancos de sangue da cidade, para explicação do programa e distribuição de folhetos de propaganda informando o seu objetivo, hora e local das reuniões. Qualquer paciente com diagnóstico definitivo ou duvidoso para doença de Chagas poderia ser encaminhado ao médico do programa. Mensalmente, 15 dias antes de cada reunião, uma acadêmica ligada ao projeto entraria em contato com a Assessoria de Comunicação Social/UEM (ASC) informando sobre a reunião. A ASC além de publicar entrevista ou nota no Boletim Informativo da Universidade, a cada 
semana antes da reunião, transmitiria a notícia para rádios e emissoras de televisão de Maringá que têm penetração inclusive estadual, para jornais do Paraná com sucursal em Maringá. Eventos como o Sesi - Ação Global eram também usados para divulgação do programa.

Agendamento dos pacientes. Os pacientes encaminhados ao Laboratório de Doença de Chagas via SUS, aqueles diagnosticados em bancos de sangue, aqueles que tinham conhecimento do programa através de propaganda em jornais, rádio e televisão ou convidados através de propaganda de boca, eram agendados obrigatoriamente para a próxima reunião após o seu contato com o Laboratório de Doença de Chagas/UEM ou com a Unidade de Psicologia Aplicada/UEM.

\section{RESULTADOS}

Perfil dos pacientes tratados. O laboratório de doença de Chagas da Universidade Estadual de Maringá mantém um projeto de atendimento ao paciente chagásico com diagnóstico, tratamento etiológico gratuito e acompanhamento clínico via Sistema Único de Saúde. Já foram atendidos 2.134 pacientes dos quais 351 apresentaram sorologia positiva para o Trypanosoma cruzi. Do total de pacientes com sorologia positiva, 131 foram tratados ou encontram-se em tratamento e serão acompanhados semestralmente por no mínimo 5 anos. São representados por $52,7 \%$ de mulheres e 47,3\% de homens. As Tabelas de 1 a 7 revelam que estes pacientes encontram-se em sua maioria $(83,8 \%)$, na faixa etária entre $31-60$ anos (Tabela 1), sendo procedentes principalmente de Minas gerais (33,1\%), São Paulo (31,5\%) e Paraná (22,3\%) (Tabela 2). Entre estes pacientes, $52 \%$ e $30,1 \%$ apresentaram alterações no eletrocardiograma e no raio-x de tórax, respectivamente (Tabelas 4,5 e 6). Quando perguntados como adquiriram a infecção chagásica, a maioria $(98,5 \%)$ respondeu ter sido através do contato com o inseto vetor (Tabela 3). Quando perguntados como descobriram que tinham a doença de Chagas, $66,2 \%$ das mulheres

Tabela 1 - Faixa etária de pacientes chagásicos dos sexos feminino e masculino atendidos no Laboratório de Doença de Chagas da Universidade Estadual de Maringá no período de1994 a 1998.

\begin{tabular}{lcccccc}
\hline $\begin{array}{l}\text { Faixa etária } \\
\text { (anos) }\end{array}$ & \multicolumn{2}{c}{ Sexo feminino } & \multicolumn{2}{c}{ Sexo masculino } & \multicolumn{2}{c}{ Total } \\
\hline $11-20$ & $\mathrm{n}^{\circ}$ & $\%$ & $\mathrm{n}^{\circ}$ & $\%$ & $\mathrm{n}^{\circ}$ & \multicolumn{1}{c}{$\%$} \\
$21-30$ & 1 & 1,4 & 0 & - & 1 & 0,8 \\
$31-40$ & 2 & 2,9 & 2 & 3,2 & 4 & 3,1 \\
$41-50$ & 14 & 20,3 & 8 & 12,9 & 22 & 16,8 \\
$51-60$ & 25 & 36,2 & 23 & 37,1 & 48 & 36,6 \\
$61-70$ & 17 & 24,6 & 21 & 33,9 & 38 & 29,0 \\
$71-80$ & 10 & 14,5 & 7 & 11,3 & 17 & 13,0 \\
Total & 0 & - & 1 & 1,6 & 1 & 0,8 \\
\hline
\end{tabular}

Tabela 2 - Procedência de pacientes chagásicos dos sexos feminino e masculino atendidos no Laboratório de Doença de Chagas da Universidade Estadual de Maringá no período de 1994 a 1998.

\begin{tabular}{|c|c|c|c|c|c|c|}
\hline \multirow[t]{2}{*}{ Estado } & \multicolumn{2}{|c|}{ Sexo feminino } & \multicolumn{2}{|c|}{ Sexo masculino } & \multicolumn{2}{|c|}{ Total } \\
\hline & $\mathrm{n}^{0}$ & $\%$ & $\mathrm{n}^{\circ}$ & $\%$ & $\mathrm{n}^{0}$ & $\%$ \\
\hline Minas Gerais & 21 & 30,9 & 22 & 35,5 & 43 & 33,1 \\
\hline São Paulo & 22 & 32,4 & 19 & 30,6 & 41 & 31,5 \\
\hline Paraná & 17 & 25,0 & 12 & 19,4 & 29 & 22,3 \\
\hline Rio Grande do Sul & 0 & - & 1 & 1,6 & 1 & 0,8 \\
\hline Estados do Nordeste & 8 & 11,6 & 8 & 12,9 & 16 & 12,3 \\
\hline Total & 68 & 100,0 & 62 & 100,0 & $130^{*}$ & 100,0 \\
\hline
\end{tabular}

* Um paciente sem informação 
Tabela 3 - Respostas de pacientes chagásicos atendidos no Laboratório de Doença de Chagas da Universidade Estadual de Maringá no período de 1994 a 1998 quando perguntados como adquiriram a doença de Chagas.

\begin{tabular}{lrrrrrrr}
\hline Via de aquisição & \multicolumn{2}{c}{ Sexo feminino } & \multicolumn{2}{c}{ Sexo masculino } & \multicolumn{2}{c}{ Total } \\
& \multicolumn{1}{c}{$\mathrm{n}^{\circ}$} & \multicolumn{1}{c}{$\%$} & \multicolumn{1}{c}{$\mathrm{n}^{\circ}$} & \multicolumn{1}{c}{$\%$} & $\mathrm{n}^{\circ}$ & \multicolumn{1}{c}{$\%$} \\
\hline Vetor & 67 & 97,1 & 62 & 100,0 & 129 & 98,5 \\
Transfusão & 1 & 1,4 & 0 & - & 1 & 0,7 \\
Não sabe & 1 & 1,4 & 0 & - & 1 & 0,7 \\
\hline Total & 69 & 100,0 & 62 & 100,0 & 131 & 100,0 \\
\hline
\end{tabular}

Tabela 4 - Resultado do eletrocardiograma e Raios-X de tórax de pacientes chagásicos atendidos no Laboratório de Doença de Chagas da Universidade Estadual de Maringá no período de 1994 a 1998.

\begin{tabular}{lccccc}
\hline Quanto à situação do & \multicolumn{2}{c}{ Alterado } & \multicolumn{2}{c}{ Normal } & \multicolumn{2}{c}{ Total } \\
& $\mathrm{n}^{\circ}$ & $\%$ & $\mathrm{n}^{\circ}$ & $\%$ & $\mathrm{n}^{\circ}$ \\
\hline Eletrocardiograma & 54 & 52,0 & 50 & 48,0 & 104 \\
Raios-X & 31 & 30,1 & 72 & 69,9 & 103 \\
\hline
\end{tabular}

Tabela 5 - Freqüência das alterações observadas no eletrocardiograma de pacientes chagásicos atendidos no Laboratório de Doença de Chagas da Universidade Estadual de Maringá no período de 1994 a 1998.

\begin{tabular}{lrr}
\hline Alteração & $\mathrm{n}^{0}$ & $\%$ \\
\hline BDASRE $^{1}$ & 1 & 1,9 \\
BIRD $^{2}$ & 3 & 5,5 \\
BRD $^{3}$ & 9 & 16,6 \\
BPIRE $^{4}$ & 1 & 1,9 \\
Bradicardia Sinusal $_{\text {Alteração de Repolarização Ventricular }}$ & 2 & 3,7 \\
Extrassístole ventricular & 5 & 9,2 \\
Sobrecarga SAE & 5 & 9,2 \\
Zona Eletricammente inativa & 1 & 1,9 \\
Associadas & & 1,9 \\
\hline Total & 1 & 48,1 \\
\hline
\end{tabular}

1BDASRE: Bloqueio da divisão ântero-superior do ramo esquerdo, ${ }^{2} \mathrm{BIRD}$ : Bloqueio Incompleto do ramo direito, ${ }^{3} \mathrm{BRD}$ : Bloqueio completo do ramo direito, ${ }^{4} \mathrm{BPIRE}$ : Bloqueio póstero-inferior do ramo esquerdo, ${ }^{5}$ Associadas: observação concomitante de duas ou três alterações acima citadas

Tabela 6 - Freqüência das alterações observadas nos Raios-X de tórax de pacientes chagásicos atendidos no Laboratório de Doença de Chagas da Universidade Estadual de Maringá no período de 1994 a 1998

\begin{tabular}{lrr}
\hline Alteração & $n^{\circ}$ & $\%$ \\
\hline Cardiomegalia & 20 & 64,5 \\
Ectasia de Aorta & 5 & 16,1 \\
Calcificação de Aorta & 1 & 3,2 \\
Alongamento de Aorta & 1 & 3,2 \\
Associadas* $^{*}$ & 4 & 12,9 \\
\hline Total & 31 & 100,0 \\
\hline
\end{tabular}

${ }^{*}$ Associadas: observação concomitante de duas ou três alterações acima citadas

e $49,2 \%$ dos homens responderam terem sentido-se mal e procurado assistência médica (Tabela 7). Por outro lado, $23,5 \%$ das mulheres e $36,1 \%$ dos homens descobriram a infecção quando foram doar sangue (Tabela 7). Durante o tratamento etiológico contra o Trypanosoma 
Tabela 7 - Respostas de pacientes chagásicos atendidos no Laboratório de Doença de Chagas da Universidade Estadual de Maringá no período de 1994 a 1998 quando perguntados como descobriram que tinham a doença de Chagas.

\begin{tabular}{|c|c|c|c|c|c|c|}
\hline \multirow[t]{2}{*}{ Situação } & \multicolumn{2}{|c|}{ Sexo feminino } & \multicolumn{2}{|c|}{ Sexo masculino } & \multicolumn{2}{|c|}{ Total } \\
\hline & no & $\%$ & no & $\%$ & $\mathrm{n}^{\circ}$ & $\%$ \\
\hline Sentiu-se mal & 45 & 66,2 & 30 & 49,2 & 75 & 58,1 \\
\hline Doação de sangue & 16 & 23,5 & 22 & 36,1 & 38 & 29,4 \\
\hline Exames de rotina & 3 & 4,4 & 3 & 4,9 & 7 & 5,4 \\
\hline Levantamento epidemiológico & 1 & 1,5 & 2 & 3,3 & 3 & 2,3 \\
\hline Teste seletivo & 0 & - & 1 & 1,6 & 1 & 0,8 \\
\hline Curiosidade & 1 & 1,5 & 1 & 1,6 & 2 & 1,6 \\
\hline Pré-operatório & 1 & 1,5 & 1 & 1,6 & 2 & 1,6 \\
\hline Perícia para aposentadoria & 1 & 1,5 & 0 & - & 1 & 0,8 \\
\hline Doação de órgãos & 0 & - & 1 & 1,6 & 1 & 0,8 \\
\hline Total & $68^{*}$ & 100,0 & $61^{*}$ & 100,0 & 129 & 100,0 \\
\hline
\end{tabular}

* Dois pacientes sem informação

cruzi, $45,2 \%$ dos pacientes apresentaram queixas relacionadas a efeitos colaterais característicos do benznidazol (ROCHAGAN ROCHE), princípio ativo do medicamento utilizado (Tabela 8).
Atendimento psico-social. O atendimento acontecia em reuniões com a presença de profissionais (psicóloga, assistente social, docente do Laboratório de Doença de Chagas) e acadêmicos de psicologia e área da saúde.

Tabela 8 - Queixas relacionadas com os efeitos colaterais do benznidazol relatadas por 45,2\% dos pacientes chagásicos tratados pelo Laboratório de Doença de Chagas da Universidade Estadual de Maringá no período de 1994 a 1998.

\begin{tabular}{ll}
\hline Efeito colateral (\%) & Queixa \\
\hline Distúrbios gerais (10,4\%) & insônia \\
dor no corpo & cefaléia \\
Distúrbios digestivos (25\%) & dor de estômago \\
anorexia & manifestações digestivas \\
Manifestações de hipersensibilidade (22,9\%) & boca amarga \\
Polineuropatia periférica (41,7\%) & alteração do paladar \\
& reações de pele \\
& dermatite \\
infecções secundárias \\
queimação nas pernas \\
\end{tabular}

Com o avançar do processo de implantação os seguintes pontos foram estabelecendo-se; com duas horas de duração, as reuniões eram divididas em duas partes: uma primeira parte informativa, específica, onde eram respondidas todas e quaisquer perguntas dos pacientes a respeito da doença de Chagas, usando vocabulário simples e explicações claras. Era também distribuído um folder que além de funcionar como propaganda e convite para a participação no programa, continha informações básicas sobre os mecanismos de aquisição da doença, sintomatologia e seu tratamento; na segunda parte da reunião era trabalhado o apoio psico-social a partir da própria fala dos membros do grupo, enfocando autoestima e cidadania. Dinâmicas de grupo, práticas 
para reflexão e relaxamento eram utilizadas. Dependendo da necessidade, segundo a avaliação da psicóloga ou da assistente social alguns pacientes eram atendidos em sessões individuais. Todo paciente era cadastrado e dados referentes a história de vida e da doença, tais como a existência de outros casos na família, o que sentiu ao receber o diagnóstico, alteração de vida após conhecimento do diagnóstico, eram anotados.

Nos trabalhos de grupo acima citados, não eram raros depoimentos como os que se seguem:

- "Eu adoro dançar, mas depois que descobrique tinha Chagas achei que tinha de ir para casa, deitar e esperar a morte..."

- "Fiquei muito triste, perdida, quando soube que tinha uma doença que não tem cura."

- "Olha, estou aqui agora, mas ninguém da minha família sabe que eu tenho esta doença"

- "Nós estamos aqui não por nós, mas pelo meu pai. Ele tem doença de Chagas e nós não temos coragem de contar a ele."

- "Ter Chagas prá mim é um problema"

- "Ter Chagas prá mim é nada, porque não posso fazer nada"

. "No meu serviço ninguém sabe"

São depoimentos que revelavam medo, insegurança, desesperança e tristeza. Além desses sentimentos, pôde ser observado que com o diagnóstico positivo os pacientes manifestavam também o peso do preconceito existente, a sensação de ser portador de doença que apresenta caráter marginalizante e estigmatizante, uma vez que de acordo com o conhecimento popular a doença de Chagas não tem cura e está relacionada à pobreza. Estes pontos eram trabalhados de forma diretiva, ou seja usando a fala dos participantes. Costumava-se fazer o aconselhamento psicológico dentro do grupo e em entrevistas individuais levando, se necessário, a um encaminhamento para psicoterapia individual ou de grupo. Em nível psicológico, o objetivo do aconselhamento era que o paciente pudesse refletir sobre sua vida lembrando que ela engloba muitos outros aspectos além da doença de Chagas.

Era comum no relato destes pacientes o depoimento da existência de outros membros da família também portadores da infecção chagásica, assim como aqueles pacientes que afirmavam nada ter mudado em suas vidas após o diagnóstico ou nada sentirem relacionado à doença de Chagas. Com o avançar da reunião ou nos encontros subseqüentes não era raro constatar-se o contrário. Era sempre possível observar um componente de conformismo e de religiosidade na fala destes pacientes. Da mesma forma, verificava-se com freqüência pacientes que entravam e permaneciam calados por todo o tempo, recusando-se, inclusive com desculpas a participar da entrevista individual.

\section{DISCUSSÃO}

O perfil clínico observado para o paciente chagásico atendido pelo Laboratório de Doença de Chagas/UEM não difere muito do descrito por outros autores em diferentes regiões do Brasil, com algumas exceções ${ }^{39}$. Diferenças maiores são encontradas com relação aos dados de Pereira e Coura $^{15}$, principalmente no que se refere a alterações no eletrocardiograma e raios- $X$ de tórax que apresentam-se menos alterado no sertão da Paraíba. Estes resultados podem estar associados a maior benignidade da doença nessa região.

Os dados que nos chamaram a atenção, por ocasião deste levantamento, foram o fato do grande número de pacientes relatarem ter descoberto a infecção após recorrerem ao banco de sangue para se candidatarem como doadores e o alto percentual de pacientes que apresentaram os incômodos efeitos colaterais decorrentes do tratamento etiológico para a doença de Chagas.

Como um dos objetivos do Laboratório de Doença de Chagas da Universidade Estadual de
Maringá é a melhoria da atenção ao paciente chagásico, estes números além de gerar mobilização para concretizar um fluxo de atendimento para candidatos a doadores de sangue com sorologia positiva para o Trypanosoma $\mathrm{Cruzi}^{13}$, levaram à criação do Programa ACHEI: Atenção ao Chagásico com Educação Integral. Este programa representaria um espaço onde o paciente chagásico, sobretudo aqueles diagnosticados em bancos de sangue e/ou em tratamento etiológico, pudesse conversar sobre doença de Chagas, conhecer seu tratamento, falar sobre seus sentimentos, atitudes e as formas de conviver com uma doença que segundo conhecimento popular não tem cura. Tal proposta baseava-se nos princípios e ações do SUS, quanto à integralidade da assistência e prevenção, vigilância epidemiológica (art. 5 e 6음 da Lei 8080/ $90)^{10}$ e nos resultados positivos que o Projeto de Apoio Psico-Social, Proaps/AMAR da própria Unidade de Psicologia Aplicada/UEM ${ }^{12}$, vinha 
obtendo com as reuniões efetuadas com grupo de portadores de doenças reumáticas dentro desta perspectiva. Aliada a estes fatos, outra idéia impulsionava a criação do programa: a tendência atual de se abordar o paciente segundo a visão do modelo humanizado de atendimento, enxergando o paciente de forma mais completa, como um ser único. Dentro desta ótica, o programa abria ainda a possibilidade de oferecer campo de estágio para acadêmicos vivenciarem a prática do modelo humanizado, além de conviver com equipe multiprofissional desenvolvendo trabalho interdisciplinar, tão importante para o atual momento de formação de profissionais da área da saúde. Vale ressaltar que, principalmente as universidades não devem esquecer que a preparação de recursos humanos constitui uma etapa indispensável da viabilização do SUS tão almejada e necessária face às características da nova abordagem do processo saúde-doença proposto.

Os sentimentos de tristeza e medo de morrer aqui detectados foram também observados por outros autores ${ }^{9}$. Se por um lado não podemos contar ainda com a realidade de fazer com que cada consulta médica seja vista como um momento privilegiado de prover o chagásico de informação científica decodificada sobre sua doença, útil para transformação e melhoria das suas condições de vida e trabalho ${ }^{8}$ o Programa ACHEl pode oferecer mais. O tipo de atividade desenvolvida neste programa além de possibilitar o esclarecimento destes pacientes sobre todos os aspectos da doença, permite que eles compartilhem com seus iguais a dúvida sobre a sua qualidade de vida após o diagnóstico, o medo, a ansiedade, o estigma, o diagnóstico positivo e a convivência com a família/grupo social; cria a oportunidade e ambiente para que cada paciente reflita sobre sua própria história, sobre o processo saúde-doença. Mesmo naqueles casos em que os pacientes recusavam-se a falar, segundo observação das profissionais da psicologia e serviço social o trabalho de grupo justificava-se, considerando que ninguém sai deste tipo de reunião como entrou.

Uma vez que uma adequada política nacional de sangue e hemoderivados são problemas ainda em aberto $^{5}$ o esclarecimento da população sobre infecções transmitidas via sangue faz-se muito importante para que ela saiba e possa exigir transfusões de boa qualidade e para o exercício da solidariedade consciente de toda a sociedade quanto à doação de sangue responsável. Além disso, não pode ser esquecido que a aquisição de informação contribui para o aumento da autoestima e exercício da cidadania.

$O$ fato de ter sido concebido dentro dos limites administrativos da universidade além de funcionar como piloto, agiliza e desburocratiza o processo. França e Abreu ${ }^{7}$ apontam que o crescimento da importância dos hospitais universitários no atendimento a pacientes chagásicos pode estar relacionado não só a maior preocupação destas instituições na realização e registros de diagnósticos como também devido a um direcionamento específico destes pacientes em virtude das pesquisas acadêmicas. Citam ainda que não pode ser descartada a idéia destes pacientes tornaremse indesejáveis para a rede contratada e mesmo filantrópica, devido a necessidade de longa permanência hospitalar e/ou necessidade de tratamentos de alto custo e baixa lucratividade.

Com relação a operacionalização da idéia, algumas observações são úteis de serem apresentadas. Este é um tipo de estratégia para educação que foi facilmente implementado em um serviço dentro da universidade. A sua viabilização junto aos serviços de saúde dependerá de decisão e disponibilidade de profissionais. Atualmente, com vários municípios brasileiros implantando o Programa de Saúde da Família (PSF) ${ }^{11}$ a idéia tornase ainda mais viável. Dentro do PSF, um grupo de atendimento psicossocial além de favorecer a integração da universidade e serviços, pode facilitar o agendamento dos pacientes que não funcionou de forma tão eficiente na experiência aqui relatada. A proposta para o futuro é fazer a reunião semanalmente, no laboratório, no dia do atendimento para coleta de sangue ou entrega de medicamento. Desta forma, será garantido o repasse das informações aos pacientes e evitará que eles se desloquem mais uma vez, já que a distância física do local das reuniões vem mostrando ser um problema.

Para os professores e profissionais envolvidos, uma vez que o programa vem estabelecendo-se como referência para informações sobre doença de Chagas, obriga-os a atualizarem-se constantemente. O apoio da imprensa propicia a divulgação e esclarecimento de toda a comunidade sobre a doença de Chagas, sua situação atual, formas de transmissão, prevenção e tratamento minorando as desinformações e preconceito. Cria também a oportunidade de coleta de dados sobre o perfil psico-social do paciente chagásico atendido no Laboratório de Doença de Chagas/UEM. Além disso com a implantação 
do Programa ACHEI abre-se a perspectiva de ampliação deste atendimento, em nível domiciliar, visando a família, direcionando o atendimento para o conceito apresentado por Gontijo et al,
$1996^{9}$ de ação integral à saúde, onde a partir de um paciente as ações são desdobradas para o grupo, com a organização de práticas preventivas coletivas e de promoção à saúde.

\section{REFERÊNCIAS BIBLIOGRÁFICAS}

1. Alcino $A B$, Guariento ME, Teixeira MAB, Lipp MEN. Avaliação psico-afetiva do paciente portador de doença de chagas crônica. Revista da Sociedade Brasileira de Medicina Tropical 26 (supl II):107, 1993.

2. Camargo ME, Silva GR, Castilho EA, Silveira AC. Inquérito sorológico da prevalência de infecção chagásica no Brasil, 1975-1980. Revista do Instituto de Medicina Tropical de São Paulo 26:192-204, 1984.

3. Coura JR, Borges-Pereira J, Alves Filho FI, Castro JAF, Cunha RV, Costa W, Junqueira ACV. Morbidade da doença de Chagas em áreas do Sertão da Paraíba e da Caatinga do Piauí. Revista da Sociedade Brasileira de Medicina Tropical 29:197-205, 1996.

4. Dias JCP. Estratégias e Perspectivas atuais de Consolidação do Controle de vetores na Etapa de Vigilância Epidemiológica. Revista da Sociedade Brasileira de Medicina Tropical 26:63-65, 1994.

5. Dias JCP. Problemas e possibilidades de participação comunitária no controle das grandes endemias no Brasil. Cadernos de Saúde Pública 14(supl. 2):19-37, 1998.

6. Dias JCP, Schofield CJ. Controle da transmissão transfusional da doença de Chagas na Iniciativa do Cone Sul. Revista da Sociedade Brasileira de Medicina Tropical 31:373-383, 1998.

7. França SB, Abreu DM. Morbidade hospitalar por doença de Chagas no Brasil. Revista da Sociedade Brasileira de Medicina Tropical 29:109-115, 1996.

8. Gontijo ED. Atendimento ao doador inapto por sorologia chagásica. Revista da Sociedade Brasileira de Medicina Tropical 29 (supl II):25-27, 1996.

9. Gontijo ED, Rocha MOC, Oliveira UT. Perfil clínicoepidemiológico de chagásicos atendidos em laboratório de referência e proposição de modelo de atenção ao chagásico na perspectiva do SUS. Revista da Sociedade Brasileira de Medicina Tropical 29:101-108, 1996.

10. Ministério da Saúde. Lei 8080 de 19-09-1990 in Conselhos Municipais de Saúde. Fórum Popular Estadual de Saúde, Série Controle Social I. Santa Catarina, 1993.

11. Ministério da Saúde/Secretaria de Políticas de Saúde. In: Anais da Reunião Técnica dos Pólos da Capacitação, Formação e Educação Permanente em Saúde da Família, 26 a 28 de maio de 1999. Brasília, 2000.

12. Mota DCGD, Borges SMR, Moretti MC, Oliveira PCG, Santos SPS, Mello AS. Desenvolvimento e intervenção: uma prática integrada de ações de saúde e psicossociais. In: Resumos do VII Encontro Regional Sul da ABRAPSO, Curitiba, p. 46, 1998.

13. Oliveira PJ, Matias GL, Silva SV, Ramos M, Cassarotti DJ, Fukushigue Y, Araújo SM. Doador de sangue com sorologia positiva para doença de Chagas no Hemocentro Maringá/PR: perfil, aconselhamento e encaminhamento. In: I Fórum de Extensão e Cultura da Universidade Estadual de Maringá. Maringá, 1996.

14. Organização Mundial de Saúde. Resolução do Conselho Execultivo da OMS. Revista da Sociedade Brasileira de Medicina Tropical 31:415-417, 1998.

15. Pereira JB, Coura JR. Morbidade da doença de Chagas em populações urbanas do sertão da Paraíba. Revista da Sociedade Brasileira de Medicina Tropical 20:101-1071987.

16. Silveira AC, Rezende DF. Epidemiologia e controle da transmissão vetorial da doença de Chagas no Brasil. Revista da Sociedade Brasileira de Medicina Tropical 27(Supl III):11-22, 1994. 